PROCEEDINGS OF THE

AMERICAN MATHEMATICAL SOCIETY

Volume 129, Number 9, Pages 2663-2669

S 0002-9939(01)05975-5

Article electronically published on February 9, 2001

\title{
DECREASING FLOW INVARIANT SETS AND FIXED POINTS OF QUASIMONOTONE INCREASING OPERATORS
}

\author{
JINGXIAN SUN AND XI'AN XU
}

(Communicated by Joseph A. Ball)

\begin{abstract}
In this paper, we obtain some new results about the existence of multiple fixed points of a kind of quasimonotone increasing operator by the new method of decreasing flow invariant set.
\end{abstract}

\section{INTRODUCTION}

Let $E$ be a real Banach space which is ordered by a cone $P$, i.e. $x, y \in E, x \leq y$ if and only if $y-x \in P . P$ is said to be a solid cone if the interior of $P$ (denoted as $\stackrel{\circ}{P}$ ) is nonempty. The dual cone of $\mathrm{P}$, denoted as $P^{*}$, is defined as the set of all continuous linear functionals $\varphi$ on $E$ with $\varphi(x) \geq 0, x \geq \theta$.

Definition 1.1 ([1]). Let $D \subset E, A: D \rightarrow E$ is called quasimonotone increasing if

$$
x, y \in D, x \leq y, \varphi \in P^{*}, \varphi(x)=\varphi(y) \Rightarrow \varphi(A x) \leq \varphi(A y) .
$$

In paper [2] Hu first studied the existence of fixed points of discontinuous quasimonotone increasing operators. Under the conditions that $A: R^{n} \rightarrow R^{n}$ is a quasimonotone increasing operator and that there are upper and lower solutions of $A, \mathrm{Hu}$ proved the existence of extremal fixed points of $A$. The paper 3 extended these results to the more general spaces such as $c_{0}, l^{p}(1 \leq p \leq+\infty)$, and so answered the open question in [2].

Recently, the author of paper [4] considered the existence of fixed points of continuous quasimonotone increasing operators in Banach spaces. Under the conditions that the cone is a regular solid cone, he proved a result about the existence of fixed points of continuous quasimonotone increasing operators. His method is by using the ordinary differential equations in Banach spaces.

In this paper, we will consider the multiple fixed points of continuous quasimonotone increasing operators in Hilbert space, which has been considered by few papers up to now. Our method is by using the decreasing flow invariant sets, which will be developed in the section 2 of this paper.

Received by the editors January 10, 2000.

2000 Mathematics Subject Classification. Primary 47H10, $47 \mathrm{H} 05$.

Key words and phrases. Quasimonotone increasing operator, fixed point, upper and lower solution.

Supported by NNSF-China and RFDP-China. 


\section{DECREASING FLOW INVARIANT SETS}

Let $H$ be a Hilbert space, $f: H \rightarrow R^{1}$ a $C^{2-0}$ functional, $f^{\prime}(x)=x-A x$ and $A: H \rightarrow H$ a quasimonotone increasing operator.

Consider the following IVP:

$$
\left\{\begin{array}{l}
\frac{d x}{d t}=A x-x, \\
x(0)=x_{0} .
\end{array}\right.
$$

By the theory of ordinary differential equations in Banach spaces, (2.1) has a unique solution, denoted by $x\left(t, x_{0}\right)$, with right maximal existence interval $\left[0, \eta\left(x_{0}\right)\right)$.

Definition 2.1. Let $M$ be a subset of $H$. If

$$
\overline{\left\{x\left(t, x_{0}\right) \mid t \in\left[0, \eta\left(x_{0}\right)\right)\right\}} \subset M, \text { for all } x_{0} \in M,
$$

then $M$ is called a decreasing flow invariant set of $f$.

Obviously, we have the following Lemma 2.1 and Lemma 2.2:

Lemma 2.1. $f\left(x\left(t, x_{0}\right)\right)$ is decreasing with $t$ on $\left[0, \eta\left(x_{0}\right)\right)$.

Lemma 2.2. The following conclusions hold:

(1) $H$ is a decreasing flow invariant set of $f$.

(2) If $\left\{M_{\mu} \mid \mu \in \Lambda\right\}$ is a family of decreasing flow invariant sets of $f$, where $\Lambda$ is an index set, then $\bigcup_{\mu \in \Lambda} M_{\mu}$ and $\bigcap_{\mu \in \Lambda} M_{\mu}$ are decreasing flow invariant sets of $f$.

(3) For any $a \in R^{1}$, the level sets $f_{a}^{\leq}$and $f_{a}^{<}$are both decreasing flow invariant sets of $f$.

Definition 2.2. Let $M$ and $D$ be decreasing flow invariant sets of $f, D \subset M$. Let $C_{M}(D)=\left\{x_{0} \mid x_{0} \in M\right.$, there exists $t^{\prime} \in\left[0, \eta\left(x_{0}\right)\right)$ such that $\left.x\left(t^{\prime}, x_{0}\right) \in D\right\} ;$

then $C_{M}(D)$ is called a decreasing flow invariant set spanned by the decreasing flow invariant set $D$. If $D=C_{M}(D)$, then $D$ is called a complete decreasing flow invariant set of $f$ relative to $M$.

Remark 2.1. Clearly, we have

(1) $H$ is a complete decreasing flow invariant set of $f$ relative to $H$;

(2) If $D_{1}, D_{2}$ are two disjoint decreasing flow invariant sets of $f$, then

$$
C_{M}\left(D_{1}\right) \cap C_{M}\left(D_{2}\right)=\emptyset \text {. }
$$

Lemma 2.3. Suppose that the connected set $M$ is a decreasing flow invariant set of $f, D$ is an open subset of $M$ and a complete decreasing flow invariant set of $f$. If $D \neq M$, then $\partial_{M} D$, the boundary of $D$ relative to $M$, is nonempty and is a complete decreasing flow invariant set of $f$.

Proof. By the connectedness and the fact that $D \neq M$, we have $\partial_{M} D \neq \emptyset$.

We first prove that $\partial_{M} D$ is a decreasing flow invariant set of $f$. For any $x_{0} \in$ $\partial_{M} D$, we consider the IVP (2.1). If there exists $t^{\prime} \in\left[0, \eta\left(x_{0}\right)\right)$ such that $x\left(t^{\prime}, x_{0}\right) \notin$ $\partial_{M} D$, then $x\left(t^{\prime}, x_{0}\right) \in D \cup\left(M \backslash \bar{D}^{M}\right)$, where $\bar{D}^{M}$ denotes the closure of $D$ relative to $M$. If $x\left(t^{\prime}, x_{0}\right) \in D$, then $x_{0} \in C_{M}(D)=D$, which contradicts $x_{0} \in \partial_{M} D$ and the fact that $D$ is an open subset of $M$. On the other hand, if $M \backslash \bar{D}^{M}$ is nonempty, $x\left(t^{\prime}, x_{0}\right) \in M \backslash \bar{D}^{M}$, noticing $M \backslash \bar{D}^{M}$ is an open set relative to $M$, by the 
continuous dependence of ordinary differential equations on initial data, we know that there exists a neighbourhood $U$ of $x_{0}$ in $M$ such that for any $x_{0}^{\prime} \in U$, there exists $t^{\prime \prime} \in\left[0, \eta\left(x_{0}^{\prime}\right)\right)$ such that $x\left(t^{\prime \prime}, x_{0}^{\prime}\right) \in M \backslash \bar{D}^{M}$. We may take $x_{0}^{\prime} \in D \cap U$ such that $x\left(t^{\prime \prime}, x_{0}^{\prime}\right) \in M \backslash \bar{D}^{M}$, which contradicts the fact $D$ is a decreasing flow invariant set. Thus $\left\{x\left(t, x_{0}\right) \mid t \in\left[0, \eta\left(x_{0}\right)\right)\right\} \subset \partial_{M} D$. Since $\partial_{M} D$ is a closed set relative to $M$, therefore $\partial_{M} D$ is a decreasing flow invariant set of $f$.

Next, we prove that $\partial_{M} D$ is a complete decreasing flow invariant set of $f$. For any $x_{0} \in M$, we suppose that there exists $t_{1} \in\left[0, \eta\left(x_{0}\right)\right)$ such that $x\left(t_{1}, x_{0}\right) \in \partial_{M} D$. Since $D$ is a complete decreasing flow invariant set, then $x_{0} \notin D$. Suppose that $x_{0} \in M \backslash \bar{D}^{M}$. Consider the following IVP:

$$
\left\{\begin{array}{l}
\frac{d x}{d t}=x-A x, \\
x(0)=x\left(t_{1}, x_{0}\right) .
\end{array}\right.
$$

Let $\bar{x}(t)$ be the unique solution of (2.2). Let $\tau=t_{1}-t$; then

$$
\begin{aligned}
\frac{d x\left(t_{1}-t, x_{0}\right)}{d t} & =\frac{d x\left(\tau, x_{0}\right)}{d\left(t_{1}-\tau\right)}=-\frac{d x\left(\tau, x_{0}\right)}{d \tau} \\
& =x\left(t_{1}-t, x_{0}\right)-A\left(x\left(t_{1}-t, x_{0}\right)\right) .
\end{aligned}
$$

By the uniqueness of the solution of (2.2), we have

$$
\bar{x}(t)=x\left(t_{1}-t, x_{0}\right), \quad 0 \leq t \leq t_{1},
$$

and $\bar{x}\left(t_{1}\right)=x\left(0, x_{0}\right)=x_{0}$. By the continuous dependence of ordinary differential equations on initial data, there exists a neighbourhood $U$ of $\bar{x}(0)$ in $M$ such that for any $x_{0}^{*} \in U$, there exists $t^{\prime} \in\left[0, \eta\left(x_{0}^{*}\right)\right)$ such that $\bar{x}^{*}\left(t^{\prime}\right) \in M \backslash \bar{D}^{M}$, where $\bar{x}^{*}(t)$ denotes the unique solution of the equation $\frac{d x}{d t}=x-A x, x(0)=x_{0}^{*}$, and $\left[0, \eta\left(x_{0}^{*}\right)\right)$ denotes the maximal existence interval of $\bar{x}^{*}(t)$. Specially, we may take $x_{0}^{*} \in U \cap D$, and consider the following IVP:

$$
\left\{\begin{array}{l}
\frac{d x}{d t}=A x-x, \\
x(0)=\bar{x}^{*}\left(t^{\prime}\right) .
\end{array}\right.
$$

In the same way as the proof of (2.3), (2.4), we have

$$
x\left(t, \bar{x}^{*}\left(t^{\prime}\right)\right)=\bar{x}^{*}\left(t^{\prime}-t\right), \quad 0 \leq t \leq t^{\prime} .
$$

Taking $t=t^{\prime}$ in (2.6), then we have $x\left(t^{\prime}, \bar{x}^{*}\left(t^{\prime}\right)\right)=\bar{x}^{*}(0)=x_{0}^{*} \in D$. Therefore, $\bar{x}^{*}\left(t^{\prime}\right) \in C_{M}(D)$. Since $C_{M}(D)=D$, then $\bar{x}^{*}\left(t^{\prime}\right) \in D$. This contradicts $\bar{x}^{*}\left(t^{\prime}\right) \in$ $M \backslash \bar{D}^{M}$. So, $x_{0} \notin M \backslash \bar{D}^{M}$ and $x_{0} \in \partial_{M} D$. The proof is completed.

Lemma 2.4. Suppose that the connected set $M$ is a decreasing flow invariant set of $f$, and $D$ is an open subset of $M$ and a decreasing flow invariant set. Then

(1) $C_{M}(D)$ is an open subset of $M$;

(2) if $C_{M}(D) \neq M, \inf _{x \in \partial_{M} D} f\left((x)>-\infty\right.$, then $\inf _{x \in \partial_{M} C_{M}(D)} f(x) \geq \inf _{x \in \partial_{M} D} f(x)$, where $\partial_{M} D$ and $\partial_{M} C_{M}(D)$ denote the boundary of $D$ and $C_{M}(D)$ relative to $M$ respectively.

Proof. By the continuous dependence of ordinary differential equations on initial data, the conclusion (1) holds. Suppose that the conclusion (2) doesn't hold; then there exists $x_{0} \in \partial_{M} C_{M}(D)$ such that $f\left(x_{0}\right)<\inf _{x \in \partial_{M} D} f(x)$. Since $f(x)$ is continuous, then there exists a neighbourhood $U$ of $x_{0}$ in $M$ such that $U \cap C_{M}(D) \neq \emptyset$ and 
for any $x_{1} \in U, f\left(x_{1}\right)<\inf _{x \in \partial_{M} D} f(x)$. In particular, we may take $x_{1} \in U \cap C_{M}(D)$ and consider the $\operatorname{IVP}(2.1)$ with initial value $x_{1}$. Then there exists $t^{\prime} \in\left[0, \eta\left(x_{1}\right)\right)$ such that $x\left(t^{\prime}, x_{1}\right) \in D$; therefore there exists $\bar{t} \in\left(0, t^{\prime}\right)$ with $x\left(\bar{t}, x_{1}\right) \in \partial_{M} D$. By Lemma 2.1, we have

$$
f\left(x\left(\bar{t}, x_{1}\right)\right) \leq f\left(x_{1}\right)<\inf _{x \in \partial_{M} D} f(x) .
$$

This is a contradiction. The proof is completed.

Theorem 2.1. Suppose that $M$ is a closed decreasing flow invariant set of $f, f$ satisfies the P.S. condition on $M$, and $\inf _{x \in M} f(x)>-\infty$. Then $f$ has at least one critical point in $M$, and $c=\inf _{x \in M} f(x)$ is a critical value of $f$.

Proof. The proof is standard and we only sketch it. Let $c=\inf _{x \in M} f(x)$. For any $n \in N$, there exists $x_{0}^{n}$ such that $c \leq f\left(x_{0}^{n}\right) \leq c+\frac{1}{n}$. Let $x\left(t, x_{0}^{n}\right)$ be the unique solution of $(2.1)$ and $\left[0, \eta\left(x_{0}^{n}\right)\right)$ be the maximal existence interval. We have

$$
\frac{d}{d t} f\left(x\left(t, x_{0}^{n}\right)\right)=\left(f^{\prime}\left(x\left(t, x_{0}^{n}\right)\right), x^{\prime}\left(t, x_{0}^{n}\right)\right)=-\left\|x^{\prime}\left(t, x_{0}^{n}\right)\right\|^{2} \leq 0 .
$$

For $0 \leq t_{1}<t_{2}<\eta\left(x_{0}^{n}\right)$, by $(2.7)$, we have

$$
\begin{aligned}
\left\|x\left(t_{2}, x_{0}^{n}\right)-x\left(t_{1}, x_{0}^{n}\right)\right\| & \leq \int_{t_{1}}^{t_{2}}\left\|x^{\prime}\left(t, x_{0}^{n}\right)\right\| d t \leq\left(\int_{t_{1}}^{t_{2}}\left\|x^{\prime}\left(t, x_{0}^{n}\right)\right\|^{2} d t\right)^{\frac{1}{2}}\left(t_{2}-t_{1}\right)^{\frac{1}{2}} \\
& \leq\left(f\left(x_{0}^{n}\right)-c\right)^{\frac{1}{2}}\left(t_{2}-t_{1}\right)^{\frac{1}{2}} .
\end{aligned}
$$

Now, we prove that $\eta\left(x_{0}^{n}\right)=+\infty$. Otherwise, if $\eta\left(x_{0}^{n}\right)<+\infty$, by (2.8), we know that $\left\|x\left(t_{2}, x_{0}^{n}\right)-x\left(t_{1}, x_{0}^{n}\right)\right\| \rightarrow 0$ as $t_{1} \rightarrow \eta\left(x_{0}^{n}\right)^{-}, t_{2} \rightarrow \eta\left(x_{0}^{n}\right)^{-}$. Thus there exists $x^{*} \in M$ such that $\lim _{t \rightarrow \eta\left(x_{0}^{n}\right)^{-}} x\left(t, x_{0}^{n}\right)=x^{*}$. Consequently, the maximal interval of existence of $x\left(t, x_{0}^{n}\right)$ would be $\left[0, \eta\left(x_{0}^{n}\right)+\delta\left(x^{*}\right)\right)$ for some $\delta\left(x^{*}\right)>0$, which is a contradiction to the maximality of $\left[0, \eta\left(x_{0}^{n}\right)\right)$. Thus, $\eta\left(x_{0}^{n}\right)=+\infty$. By (2.7), for any $t>0$, we have

$$
\int_{0}^{t}\left\|f^{\prime}\left(x\left(t, x_{0}^{n}\right)\right)\right\|^{2} d t=f\left(x\left(0, x_{0}^{n}\right)\right)-f\left(x\left(t, x_{0}^{n}\right)\right) \leq f\left(x_{0}^{n}\right)-c<+\infty .
$$

Consequently, there exist $t_{n} \in\left\{x\left(t, x_{0}^{n}\right) \mid t \in\left[0, \eta\left(x_{0}^{n}\right)\right)\right\}, t_{n} \rightarrow+\infty$, such that

$$
c \leq f\left(x\left(t_{n}, x_{0}^{n}\right)\right) \leq f\left(x_{0}^{n}\right) \leq c+\frac{1}{n},\left\|f^{\prime}\left(x\left(t_{n}, x_{0}^{n}\right)\right)\right\| \leq \frac{1}{n} .
$$

Then, by the P.S. condition, we know the conclusion holds. The proof is completed.

By Lemma 2.3 and 2.4, we have the following Theorem 2.2.

Theorem 2.2. Suppose that the connected set $M$ is a decreasing flow invariant set of $f$, and $D$ is an open subset of $M$ and is also a decreasing flow invariant set. Then $M$;

(1) $C_{M}(D)$ is a decreasing flow invariant set and $C_{M}(D)$ is an open subset of

(2) if $C_{M}(D) \neq M$, then $\partial_{M} C_{M}(D)$ is a complete decreasing flow invariant set;

(3) if $C_{M}(D) \neq M$ and $\inf _{x \in \partial_{M} D} f(x)>-\infty$, then $\inf _{x \in \partial_{M} C_{M}(D)} f(x) \geq \inf _{x \in \partial_{M} D} f(x)$. 
For applications in the sequel, we give the following similar result.

Theorem 2.3. Suppose that the connected set $M$ is a decreasing flow invariant set of $f, D$ is a closed subset of $M, \stackrel{\circ}{D} \neq \emptyset(\stackrel{\circ}{D}$ denotes the interior of $D$ relative to $M)$, and $D$ is also a decreasing flow invariant set. Also suppose that $f$ satisfies the P.S. condition on $\partial_{M} D$ and has no critical point on $\partial_{M} D$ and $\inf _{x \in \partial_{M} D} f(x)>-\infty$. Then the conclusions (1)-(3) in Theorem 2.2 hold.

Proof. We only need to prove that $C_{M}(D)$ is an open subset of $M$. For any $x_{0} \in$ $C_{M}(D)$, it is easy to prove that there exist $t^{\prime} \in\left[0, \eta\left(x_{0}\right)\right)$ such that $x\left(t^{\prime}, x_{0}\right) \in \stackrel{\circ}{D}$. Then by the continuous dependence of ordinary differential equations on initial data, we know that $C_{M}(D)$ is an open subset of $M$. The proof is completed.

Remark 2.2. The main results in this section come from [7].

\section{FiXed POINTS OF QUASIMONOTONE INCREASING OPERATORS}

In this section, we will discuss the multiple fixed points of quasimonotone increasing operator.

Lemma $3.1([5])$. Let $P$ be a cone in $E$, and also a distance set. Assume that

(1) $u, v \in C^{1}\left[\left[t_{0}, t_{0}+a\right), E\right], f \in C\left[\left[t_{0}, t_{0}+a\right) \times E, E\right]$ and $f(t, x)$ is quasimonotone increasing in $x$ for each $t \in\left[t_{0}, t_{0}+a\right)$;

(2) $u^{\prime}(t)-f(t, u(t)) \leq v^{\prime}(t)-f(t, v(t)), \quad t \in\left[t_{0}, t_{0}+a\right)$;

(3) $\|f(t, x)-f(t, y)\| \leq g(t,\|x-y\|), x \in E \backslash P, y \in \partial P$, where $g$ is a uniqueness function.

Then $u\left(t_{0}\right) \leq v\left(t_{0}\right)$ implies that $u(t) \leq v(t), t \in\left[t_{0}, t_{0}+a\right)$.

Now, let us formulate some conditions.

$\left(H_{1}\right)$ Let $H$ be a Hilbert space, $P \subset H$ a solid cone, and $f: P \rightarrow R^{1}$ a $C^{2-0}$ functional. Suppose that $f^{\prime}(x)=x-A x, A: P \rightarrow P$ is quasimonotone increasing, and $f$ satisfies the P.S. condition on $P$.

$\left(H_{2}\right)$ There exist $x_{0} \in \stackrel{\circ}{P}, y_{0} \in \stackrel{\circ}{P}$ such that $A x_{0} \leq x_{0}, A y_{0} \geq y_{0}$.

Remark 3.1. Under the condition $\left(H_{1}\right)$, by Lemma 3 in [6], we know that $P$ is a decreasing flow invariant set.

Theorem 3.1. Suppose that $\left(H_{1}\right),\left(H_{2}\right)$ hold and that

(1) $y_{0} \not \leq x_{0}$;

(2) $\inf _{x \in D_{1}} f(x)>-\infty, f$ has no critical points on $\partial_{P} D_{1}$, where $D_{1}=\left[\theta, x_{0}\right]$.

Then $A$ has at least two fixed points on $P$.

Proof. Let $D_{2}=\left\{x \in P: x \geq y_{0}\right\}$. Since $H$ is a Hilbert space and $P$ is a closed convex set, we know that $P$ is a distance set. It follows from Lemma 3.1 that $D_{1}$ and $D_{2}$ are two decreasing flow invariant sets of $f$. Since $y_{0} \not \leq x_{0}$, then $D_{1} \cap D_{2}=\emptyset$. By the definition of $C_{P}\left(D_{1}\right)$, we know that $C_{P}\left(D_{1}\right) \cap D_{2}=\emptyset, C_{P}\left(D_{1}\right) \neq P$. It follows from Theorem 2.3 that $\partial_{P} C_{P}\left(D_{1}\right)$ is a decreasing flow invariant set and that

$$
c_{1}=\inf _{x \in \partial_{P} C_{P}\left(D_{1}\right)} f(x) \geq \inf _{x \in \partial_{P} D_{1}} f(x)>-\infty .
$$

Let $c_{2}=\inf _{x \in D_{1}} f(x)$. By Theorem 2.1, $c_{1}, c_{2}$ are critical values and there exist $x_{1}, x_{2}$ 
such that

$$
x_{1} \in \partial_{P} C_{P}\left(D_{1}\right), \quad x_{2} \in D_{1} ; \quad f\left(x_{i}\right)=c_{i}, \quad i=1,2 .
$$

Clearly, $x_{1}, x_{2}$ are two fixed points of $A$ on $P$. The proof is completed.

Theorem 3.2. Suppose that $\left(H_{1}\right),\left(H_{2}\right)$ hold and that

(1) $y_{0} \leq x_{0}$;

(2) there exists a $z_{0} \in D_{2}$ with $z_{0} \not \leq x_{0}$ such that

$$
\max \left\{f(\theta), f\left(z_{0}\right)\right\}<\inf _{x \in \partial_{P}\left(D_{1} \cap D_{2}\right)} f(x),
$$

where $D_{1}=\left[\theta, x_{0}\right], D_{2}=\left\{x \in P \mid x \geq y_{0}\right\}$;

(3) $f$ has no critical points on $\partial_{P}\left(D_{1} \cap D_{2}\right) ; \inf _{x \in D_{1} \cup D_{2}} f(x)>-\infty$.

Then $A$ has at least five fixed points.

Proof. Let $D_{3}=D_{1} \cap D_{2}$. It follows from Lemma 3.1 that $D_{1}$ and $D_{2}$ are two decreasing flow invariant sets of $f$. Thus $D_{3}$ is also a decreasing flow invariant set. Then, by (3.1), we easily prove that $\theta \notin C_{D_{1}}\left(D_{3}\right), z_{0} \notin C_{D_{2}}\left(D_{3}\right)$. It follows from Theorem 2.3 that $\partial_{D_{1}} C_{D_{1}}\left(D_{3}\right), \partial_{D_{2}} C_{D_{2}}\left(D_{3}\right)$ are two decreasing flow invariant sets of $f$.

Let $F_{1}=D_{1} \backslash C_{D_{1}}\left(D_{3}\right), F_{2}=D_{2} \backslash C_{D_{2}}\left(D_{3}\right)$. It is easy to prove that $F_{i}(i=1,2)$ are decreasing flow invariant sets of $f$.

Let $c_{1}=\inf _{x \in \partial_{D_{1}} C_{D_{1}}\left(D_{3}\right)} f(x), \quad c_{2}=\inf _{x \in \partial_{D_{2}} C_{D_{2}}\left(D_{3}\right)} f(x), \quad c_{3}=\inf _{x \in F_{1}} f(x), c_{4}=$ $\inf _{x \in F_{2}} f(x), \quad c_{5}=\inf _{x \in D_{3}} f(x)$. By Theorem 2.1, we know that $c_{i}(i=1,2,3,4,5)$ are critical values, and there exist five points $x_{i}(i=1,2,3,4,5)$ such that $f\left(x_{i}\right)=c_{i}$. Clearly, $x_{i}(i=1,2,3,4,5)$ are five fixed points of $A$. The proof is completed.

Remark 3.2. In Theorems 3.1 and 3.2, we give the results on the multiple fixed points of a quasimonotone increasing operator under the condition that the lower solutions may not be less than the upper solutions, which is different from all the known results on the fixed points of a quasimonotone increasing operator. Our method is also different from all the known results.

Remark 3.3. Clearly, our results can be applied to the case when $A$ is increasing.

Example. Let $H=R^{2}, P=R^{+} \times R^{+}$. Consider the mapping $A: R^{2} \rightarrow R^{2}$,

$$
A\left(\left(x_{1}, x_{2}\right)\right)=\left(\frac{1}{8}\left(1+\sin x_{1}\right)+\frac{1}{4} x_{2}, \frac{1}{4} x_{1}+5 \pi\left(1+\cos x_{2}\right)\right), \quad\left(x_{1}, x_{2}\right) \in R^{2} .
$$

It is easy to see that $A: P \rightarrow P$ is a quasimonotone increasing operator. Let $f\left(x_{1}, x_{2}\right)=\frac{1}{2}\left(x_{1}^{2}+x_{2}^{2}\right)-g\left(x_{1}, x_{2}\right), g\left(x_{1}, x_{2}\right)=\frac{1}{8} x_{1}-\frac{1}{8} \cos x_{1}+\frac{1}{4} x_{1} x_{2}+5 \pi x_{2}+$ $5 \pi \sin x_{2}$. Then we have

$$
\begin{aligned}
\left(\frac{\partial g\left(x_{1}, x_{2}\right)}{\partial x_{1}}, \frac{\partial g\left(x_{1}, x_{2}\right)}{\partial x_{2}}\right) & =\left(\frac{1}{8}\left(1+\sin x_{1}\right)+\frac{1}{4} x_{2}, \frac{1}{4} x_{1}+5 \pi\left(1+\cos x_{2}\right)\right) \\
& =A\left(\left(x_{1}, x_{2}\right)\right)
\end{aligned}
$$




$$
\begin{aligned}
\left(\frac{\partial f\left(x_{1}, x_{2}\right)}{\partial x_{1}}, \frac{\partial f\left(x_{1}, x_{2}\right)}{\partial x_{2}}\right) & =\left(x_{1}, x_{2}\right)-\left(\frac{\partial g\left(x_{1}, x_{2}\right)}{\partial x_{1}}, \frac{\partial g\left(x_{1}, x_{2}\right)}{\partial x_{2}}\right) \\
& =x-A x
\end{aligned}
$$

where $x=\left(x_{1}, x_{2}\right)$. Let $x_{0}=\left(\frac{\pi}{2}, \pi\right), y_{0}=(\pi, 8 \pi)$. Then we have

$$
\begin{aligned}
A x_{0} & =\left(\frac{1}{8}\left(1+\sin \frac{\pi}{2}\right)+\frac{1}{4} \pi, \frac{1}{8} \pi+5 \pi(1+\cos \pi)\right) \\
& =\left(\frac{1}{4}+\frac{\pi}{4}, \frac{1}{8} \pi\right)<\left(\frac{\pi}{2}, \pi\right)=x_{0}, \\
A y_{0} & =\left(\frac{1}{8}(1+\sin \pi)+2 \pi, \frac{1}{4} \pi+5 \pi(1+\cos 8 \pi)\right) \\
& =\left(\frac{1}{8}+2 \pi, 10.25 \pi\right)>(\pi, 8 \pi)=y_{0} .
\end{aligned}
$$

It is easy to see that $y_{0}>x_{0}$, and so $y_{0} \not \leq x_{0}$. We also easily know that $f\left(x_{1}, x_{2}\right)>$ $-\frac{9}{8},\left(x_{1}, x_{2}\right) \in\left[0, \frac{\pi}{2}\right] \times[0, \pi]$. Therefore $\inf _{x \in\left[\theta, x_{0}\right]} f(x)>-\frac{9}{8}$. It is easy to check that

$$
A\left(\frac{\pi}{2}, y\right) \neq\left(\frac{\pi}{2}, y\right), \quad A(x, \pi) \neq(x, \pi), 0 \leq x \leq \frac{\pi}{2}, 0 \leq y \leq \pi .
$$

So, $f$ has no critical point on the boundary of $\left[0, \frac{\pi}{2}\right] \times[0, \pi]$. By Theorem 3.1, we know that $A$ has at least two fixed points on $P$.

\section{ACKNOWLEDGMENT}

The authors wish to express their sincere gratitude to the reviewer for his/her helpful suggestion in improving the paper.

\section{REFERENCES}

[1] P.Volkmann, Gewöche, Differentiallungleichungen mit quasimonoton wachsenden Funktionen in topogischen Vektorräumen, Math. Z. 127 (1972), 157-164. MR 46:7661

[2] S.Hu, Fixed points for discontinuous quasimonotone maps in $R^{n}$, Proc. Amer. Math. Soc. 104 (1988), 1111-1114. MR 89k:47093

[3] S.Schmidt, Fixed points for discontinuous quasimonotone increasing maps in sequence spaces, Proc. Amer. Math. Soc. 115 (1992), 361-363. MR 93c:47072

[4] Gerd Herzog, A fixed point theorem for quasimonotone increasing mappings, Acta Sci. Math. (Szeged) 64 (1998), 293-297. MR 99g:47132

[5] Dajun Guo and V.Lakshmikantham, Nonlinear Problems in Abstract Cones, Academic Press.Inc., Boston, 1988. MR 89k:47084

[6] Jingxian Sun, The Schauder condition in critical point theory, Chinese Sci. Bull. 31 (1986), 1157-1162. MR 88a:58038

[7] J. Sun, On Some Problems about Nonlinear Operators, Ph.D. Thesis, Shandong University, Jinan, 1984(in chinese).

Department of Mathematics, Shandong University, Jinan, Shandong, 250100, People's Republic of China

Department of Mathematics, Shandong University, Jinan, Shandong, 250100, People's Republic of China

E-mail address: xxa@math.sdu.edu.cn 